Boise State University

ScholarWorks

Psychological Sciences Faculty Publications and

Presentations

9-1-2013

Factors and Convergent Validity of the Pet Attachment and Life Impact Scale (PALS)

Lisa DeMarni Cromer

University of Tulsa

M. Rose Barlow

Boise State University 


\title{
Factors and Convergent Validity of The Pet Attachment and Life Impact Scale (PALS)
}

\author{
Lisa DeMarni Cromer \\ Department of Psychology, University of Tulsa \\ M. Rose Barlow \\ Department of Psychology, Boise State University
}

\begin{abstract}
Human-animal interaction (HAI) provides benefits for humans. Emotional attachment to pets is a possible mechanism for benefits but there is no standard operationalization for "attachment to pets." The study presented here $(N=651)$ uses a pet attachment measure based on qualitative research about benefits of pets. This measure, the Pet Attachment and Life Impact Scale (PALS), has four factors that measure Love, Regulation, Personal Growth, and Negative Impacts. We present exploratory factor analysis and confirmatory factor analysis of the instrument. We then examine convergent validity with four a priori derived measures of pet attachment (Anthropomorphism Scale, CENSHARE PAS, CABS, LAPS) and a social support scale. We provide evidence that having a current relationship with a pet is related to higher scores on the PALS than having a former pet relationship, evidencing that the PALS is a relational measure. Overall, females are more attached to pets than are males, and dog owners are most attached, followed by cat owners and owners of other pets.
\end{abstract}

Key words: Attachment, Pets, Measurement

Acknowledgements: The authors thank Daniel Simonet at the University of Tulsa for statistical consultation. Funding provided by The University of Tulsa faculty summer fellowship.

Factors and Convergent Validity of the Pet Attachment and Life Impact Scale (PALS)

Pets are an important aspect of Western culture and almost half of dog or cat owners consider pets to be members of the family (American Veterinary Medicine Association [AVMA], 2006). Examining potential benefits of pet ownership is a growing area of applied research. In the literature, there are increasing numbers of publications related to humananimal interactions. This trend was evidenced in a recent EBSCO ${ }^{\circledR}$ search of PsycINFO, MEDLINE, CINAHL, SocINDEX, and Education Research Complete search engines using the terms "pet attachment" or "pets" or "human animal interaction." The total publications for each period (for articles published in English with human subjects) consistently increased: 1971 - 1980: 588 publications; 1981 - 1990: 1,511; 1991 - 2000: 5,347; 2001 - 2010: 14,510 publications. Despite researchers' focus on benefits of pets, little is understood about our attachment to pets. In fact, when attachment to pets is reviewed in the literature, the term attachment is typically used interchangeably with attitudes towards pets (Herzog, 2007). The current study introduces a scale that has been developed to measure positive and negative aspects of relationships with pets, including the impact of pets on 
owners; it also operationalizes attachment based on the attachment literature.

\section{Benefits of Pets}

A theme in human-animal interaction (HAI) literature is that animals can positively impact human physical and psychological health, as well as mitigate stressors that lead to long term health problems. HAI can reduce blood pressure (Allen, 2003) and frequency of doctor visits (Headey, 1999), and is associated with increased life expectancy (Allen, Shykoff, \& Izzo, 2001; Friedmann, Thomas, Wilson, \& Turner, 1995). Pet owners may have a decreased risk of cardiovascular disease even when they eat more meat and more fast food than non-pet owners (Rowan \& Beck, 1994). Interaction with an animal may also directly and positively influence humans' physiological state, which is related to increases in owners' oxytocin levels when their pets gaze at them (Nagasawa, Mogi, \& Kikusui, 2009).

There also appear to be psychological benefits of pet ownership. Siegel and colleagues (Siegel, Angulo, Detels, Wesch, \& Mullen, 1999) found that pet ownership was associated with less depression in AIDS victims. Unfortunately, the reasons for benefits from HAI are speculative. It may be that HAI is a substitute for human attachment and social support, and that pet attachment increases in the face of a stressor. Alternately, it may be that pets are merely an addition to human social support networks (Stammbach \& Turner, 1999).

The existing HAI research is methodologically limited because it has often been conducted using convenience samples and because HAI has been inconsistently operationalized. Thus, cross-study comparison is difficult. For example, studies have examined pet attachment (Johnson, Garrity, \& Stallones, 1992), anthropomorphism (Antonacopoulos \& Pychyl, 2008), and other, undefined aspects of having a companion animal. Furthermore, it may be that pet attachment, not pet ownership, is important. Unfortunately, even scales that attempt to capture a relational or attachment value of pets do not clearly define the nature of human-animal relationships.

\section{Social Support and Pets}

It appears that pets may fill a relational void in some owners' lives. Social support has been examined in several studies, but findings have been inconsistent. Staats, Wallace, and Anderson (2008) found that pets helped bridge the gap between family life change and new social networks in college life by providing support for coping with stress, even for students who lived at home while attending college. In one study, authors speculated that dogs may be a source of extra social support for people who already had enough skills and resources to have sufficient human social support. For people with low levels of social support from humans, dogs may not provide enough support to compensate for the overall lack of support in peoples' lives (Antonacopoulos \& Pychyl, 2010). Whether a pet serves as a source of support may depend on aspects of the humananimal relationship. Zilcha-Mano, Mikulincer, and Shaver (2011) found that an insecure human-pet relationship was related to owners' negative attitudes about their pets. In this study, participants' attachment deficits to humans were consistent with deficits in attachment to pets.

Sable (1995) reported that pets served as a replacement for, or an extension of, attachment to humans. Nonetheless, the hypothesis about relational need is the subject of some disagreement. Cohen (2002) found that people who were more attached to pets were no more likely to lack close human relationships, although pets met both intimacy and affection needs. Kurdek (2009) found that students who had high levels of attachment to their dogs had 
similar attachment levels in human relationships. One strength of the research conducted by Kurdek is that he operationalized attachment based on attachment theory, and attachment bonds were evaluated based on criteria used in human attachment studies. Because no measure at the time operationalized pet attachment in this way, Kurdek used open-ended questions to evaluate attachment relationships. Kurdek based analyses on Mary Ainsworth's model of human parent-child attachment relationships (Ainsworth, 1991), and found that pets served some of the same key functions of attachments that Ainsworth had observed in parent-child dyads: proximity maintenance, separation distress, secure base, and safe haven (Kurdek, 2009, p. 360). However, he found that dogs were not strongly associated with a safe haven for study participants. Although this study sample was limited to dog owners, it shed some light on attachment behaviors because it queried relational aspects of attachment, such as who participants turned to for comfort, rather than asking about beliefs or attitudes about dogs.

Researchers have also examined the construct of anthropomorphism as a means of operationalizing HAI. Anthropomorphism is a way of relating to non-human animals by giving them human or human-like characteristics. It may be that people who highly anthropomorphize their pets are attempting to use the pet as a human-like source of support. This reasoning is consistent with findings that pet owners with low perceived social support from other humans engaged in more anthropomorphizing of their pet dogs than did pet owners with higher perceived social support. Not surprisingly, perceived social support from the dog itself was associated with anthropomorphizing the dog (Antonacopoulos \& Pychyl, 2008).

Some investigators have queried whether men and women benefit differently from HAI. In a review of the literature, Herzog (2007) found that men and women are similar on level of attachment to pets; studies in which women were more attached had small effect sizes. Staats, Sears, and Pierfelice (2006) found that, while men and women were equally as likely to have and to value pets, women were more likely than men to report social support reasons for having a companion animal, such as reducing loneliness and having emotional support through hard times. Men were more likely to report keeping a companion animal for pragmatic reasons, such as running (exercise) and hunting.

Overall there is conflicting evidence about what predicts beneficial HAI (Nicoll, Trifone, \& Samuels, 2008). Inconsistent findings across the literature are likely affected by small and biased (e.g., veterinary students as subjects) samples (Herzog, 2011). HAI relationships are complex, and existing measures are limited by what aspects of HAI are measured (e.g., Morovati, Steinberg, Taylor, \& Lee, 2008; Winefield, Black, \& Chur-Hansen, 2008). These factors limit the interpretation and generalizability of findings, especially when planning future intervention work. Understanding the important aspects of different meanings and benefits of HAI will help researchers design therapy interventions. For example, understanding the factors in HAI that provide benefits would offer insight to whether therapy animals can be beneficial when there is no meaningful relationship between the human and animal.

\section{Development of the Pet Attachment and Life Impact Scale}

The Pet Attachment and Life Impact Scale (PALS) was developed to address several limitations in the literature, in order to add to the understanding of who receives what benefits from HAI. At the time of the scale development, the shortcomings in the literature included: (1) Biased sampling (e.g., recruitment from veterinary schools); (2) Not examining 
differences in HAI as they related to type of pet; and (3) Inconsistent operationalization of pet attachment (Crawford, Worsham, \& Swinehart, 2006). These limitations still persist. Our aim was to qualitatively derive a measure of HAI and attachment to pets in order to understand a broader spectrum of human-pet relationships in a sample that was recruited from a source other than a pet store, pet shelter, pet adoption agency, or veterinary school.

The PALS is unique in that it was derived through textual analysis. In 2003, we surveyed 350 undergraduates who participated in a human subjects pool (HSP). We asked them "How do you think having pets impacted your life?” The common themes and statements, in the language of the students, were used to derive a scale that we named based on face validity: The Pet Attachment and Life Impact Scale (PALS). This name reflected the nature of the items which indicated that pets impacted lives in positive and negative ways (life impact) and other items inferring that pets were a source of comfort and security (attachment). We used the term "attachment" because it appeared that pets served a relational and emotional attachment for humans. The items that we developed for the measure were based on statements participants offered, and we noted that these mirrored attachment relationships. It is important to note that this scale development occurred before Kurdek had published animal attachment papers, and likely represents a zeitgeist of animal attachment. From respondents' answers, our textual analysis suggested that aspects of attachment included a secure base, source of comfort (emotion regulation), and love-all of which were consistent with the original definition of attachment offered by Bowlby (1982).

We called it the PALS-30 because it consisted of the 30 most common statements provided by our participants. We presented these findings at The American Academy for the
Advancement of Science Conference in Seattle, Washington (Cromer \& Freyd, 2004). Subsequent data collection used this measure and included the question "Is there anything else you want to tell us about how pets are important to you?" Most people responded to this question, and we drew on these responses for further measure development. This most recent version of the PALS has good psychometric properties (presented in this paper) that will help elucidate the complex roles pets can play in our lives.

\section{Goals of the Current Study}

The goal of the current study was to evaluate the psychometric properties of the PALS using principle axis factoring and confirmatory factor analysis. Validity was examined through comparison with other measures of pet attachment and with measures of social support from humans. A consistent theme in other published scales is a focus on positive aspects (particularly attachment) in pet ownership. Other measures generally do not query potentially negative aspects of pet ownership such as financial or time costs incurred from pet care, which could contribute additional stressors that could negatively impact the pet owner. In the measure we developed, we also included potentially negative aspects of how pets could impact one's life.

We examined whether pet attachment reflected attitudes or relationships by assessing differences in attachment based on whether one currently owned a pet. We expected that if the PALS was a relational measure of current attachment and relationship satisfaction, then current owners would have higher scores than former pet owners. Conversely, we expected that if the PALS was measuring constructs related to cognitions and beliefs about pets, then owners and former owners would not differ on the scale. We also examined attachment as it 
related to type of pet and determined whether there were systematic individual differences in pet attachment, such as gender of participants.

In the current study, we used a relatively unbiased sample of participants through a human subjects pool. The benefit of a research pool is that participants' motivation for participating is to obtain research credit; other research in this area may be biased because participants may be motivated to participate in a study in order to show support for the notion that pet ownership is beneficial. We invited people who had ever lived with a pet, whether or not they owned the pets, to respond to the questionnaire. People who did not have pets were allowed to skip any of the animal questionnaires that did not apply to them (while still answering questions about human social support). The new measure is the Pet Attachment and Life Impact Scale (PALS; Cromer \& Freyd, 2004).

\section{Study Questions and Hypotheses}

The first goal of the study was to conduct an exploratory factor analysis (EFA) of the PALS, followed by a confirmatory factor analysis (CFA), in order to see whether the hypothesized factors from the EFA are supported in a second sample. We then examined convergent validity with four measures of pet attachment that were most frequently used in peer-reviewed manuscripts. Given the mixed literature on the function of pet attachment as a means of providing social support, we examined the subscales of the PALS in order to determine whether particular aspects of pet attachment (as indexed by the subscales) are related to perceived human social support. We predicted that females would have higher PALS scores than males. We also examined whether species of peoples' pets related to their attachment to pets.
A test of criterion validity was conducted to evaluate whether the PALS was sensitive to differences in current relationship attachment and current relational benefits of pets. Kurdek (2009) had attempted to evaluate this idea by wording questions in the present tense "Who do you turn to," rather than "Who would you turn to" to order to capture actual and current aspects of emotional reliance on pets instead of possible sources of emotional reliance. The PALS has many items that specify current emotional reliance and engagement (e.g., item 26, "My pet calms me down.”). Thus, the PALS should be sensitive to whether one currently has a pet that provides emotional regulation. In contrast, if the PALS merely was measuring attitudes or beliefs about pets, then temporal proximity of relationship to a pet would show differences on PALS subscales. In other words, we expected that people who were living with a pet would have higher levels of attachment than those who previously lived with pets but who were not currently living with pets. Differences between these two groups on the subscales would suggest that each of the subscales of the PALS is measuring a current relational value of having a pet, whereas no differences between the groups would suggest that the PALS is not sensitive to emotional reliance but rather to beliefs about the value of pet ownership.

\section{Method}

\section{Participants}

Participants were 651 college students (51.78\% female) at a northeastern university who participated in the study for partial fulfillment of course credit for an introductory psychology class. The mean age was 19 years $(S D=1.9$, range $=18-47)$. They identified as 49\% ( $n=328)$ White/Non-Hispanic, 20\% ( $n=$ 134) White/Hispanic, 8\% $(n=56)$ African American, 18\% ( $n=118)$ Asian, 1\% $(n=7)$ Native American, and .1\% ( $n=1)$ Other. Of our 
participants, $41 \%$ currently lived with a pet, 30\% were not currently living with a pet but had lived with a pet in the last year, $22 \%$ had not lived with a pet in the last year but had lived with a pet at some point in their lives, and $7 \%$ had never lived with a pet. The type of pet about which respondents completed the PALS was queried with an open-ended question. These resulted in seven pet categories: dog, cat, bird, reptile, fish, rodent, and farm animal.

\section{Measures}

We included the most common and beststudied measures of HAI, drawing from previous literature.

Anthropomorphism Scale (Albert \& Bulcroft, 1988) is rooted in theories of family development. At the time of this writing, the original manuscript had been cited 167 times in Google Scholar. Qualitative and quantitative interviews with pet owners and non-owners formed the basis of the scale. People with dogs were more likely to anthropomorphize their pets than were people with cats or other types of animals. Owners who were remarried or who were single (never-married or divorced - but not widowed), and people with no children were more likely to anthropomorphize their pets (Albert \& Bulcroft, 1988). The items examine feelings towards pets, rights of pets, and how much the subject would sacrifice for a pet. The Anthropomorphism Scale has ten items that measure the degree to which participants interpret their dogs' behaviors and attributes as having human-like traits. The full scale score is a sum of responses with possible score range: 7 39. Cronbach's alpha in the original study is .69. In the current sample, Cronbach's alpha $=$ .68.

CENSHARE Pet Attachment Scale (PAS; Holcomb, Williams, \& Richards, 1985) has 27 items derived a priori based on human attachment styles and other animal attachment questionnaires. At the time of this writing, the original manuscript had been cited 57 times in Google Scholar. The PAS measures attachment to conventional pets. There are two factors: Relationship Maintenance, i.e., "behaviors broadly related to physical and sensual interaction; communication; time, and financial investment” (p. 29), and Intimacy, i.e., “attitudes surrounding emotional importance; physical proximity; planning for close physical proximity" (p. 31). It queries human-pet interactions such as grooming and playing with pet, relationship aspects such as confiding in a pet, and discipline such as hitting pet if the pet misbehaves. Responses are provided on a 4point scale. In previous research, scores on each scale were negatively correlated with size of household. Females scored higher on both subscales than did males (Holcomb et al., 1985). A limitation of this study is the biased recruitment from a single site (potentially limiting demographic factors), where pet owners visited for routine pet health care, thus results may not generalize to the values and attachments of a wider population of pet owners. In the present study, we reverse scored this scale so that positive correlations indicated agreement with other measures of pet attachment. Cronbach's alpha in the original publication (Holcomb et al., 1985) was .83 for Relationship Maintenance and .74 for Intimacy. In the current data set, Cronbach's alpha for Relationship Maintenance is .88 , and .78 for Intimacy.

Companion Animal Bonding Scale (CABS; Poresky, Hendrix, Mosier, Samuelson, 1987) is an 8-item, face valid (Anderson, 2007) instrument that assesses child-pet activities, such as frequency of caring for and sleeping in the same room as a pet. At the time of this writing, the original manuscript had been cited 35 times in Google Scholar. Responses are provided on a 5 -point scale where $5=$ Always and $1=$ Never . Poresky and colleagues (1987) reported Cronbach's alpha $=.77$; in the current sample 
Cronbach's alpha $=.86$. The scale has good reliability in college students and good construct validity in a sample of 121 high school and college students. The authors operationalized bonding with a pet by querying caretaking behaviors such as being responsible for pet care, and holding or stroking the pet.

Lexington Attachment to Pets Scale (LAPS; Johnson et al., 1992) is a 23-item measure of attachment to dogs and cats. Responses are provided on a 4-point scale where $1=$ Disagree strongly and $4=$ Agree strongly. At the time of this writing, the original manuscript had been cited 81 times in Google Scholar. The authors developed the LAPS as a compendium of items from existing measures of pet attachment, and included a priori derived items as well. The authors used a random telephone survey of metropolitan-based participants who identified as being at least 18 years old and owning at least one pet (91\% were dog or cat owners). People with higher attachment scores were female, black, older, less well-educated, had lower income, and came from smaller households. This measure has three subscales: General attachment, People substitution, and Animal rights and welfare. The full scale alpha was .93 (Johnson et al., 1992).

Pet Attachment and Life Impact Scale. The development of the PALS is described above. The 39-item version is used in the current study. Responses are provided on a 5point Likert scale: $1=$ Not at all; $2=$ Somewhat, $3=$ Moderately, $4=$ Quite $a$ bit, and $5=$ Very much.

The Multidimensional Scale of Perceived Social Support (MSPSS; Zimet, Dahlem, Zimet, \& Farley, 1988) is a 12-item measure that offers subjective assessment of perceived social support from 3 distinct subgroups: family, friends, and a significant other. The scale is reliable over a time scale of 2 - 3 months (testretest $r=.85$; Zimet et al., 1988). Cronbach's alpha of .88 indicates that the scale is internally consistent (Zimet et al., 1988). It has a negative correlation with depression and anxiety. Women tend to score higher than men on perceived social support from significant other and from friends, though there is little gender difference in perceived support from family (Zimet et al., 1988). In previous research, the MSPSS has shown some relationship with pet attachment, though the interaction may be complex (Antonacopoulos \& Pychyl, 2010).

\section{Procedure}

The investigators obtained IRB approval prior to data collection. Data collection was anonymous, using a feature of the Sona System $^{\mathrm{TM}}$ software. The study took fewer than 30 minutes for students to complete, and all data were collected over a single semester.

Principle axis factoring (PAF) with promax rotation in SPSS $^{\mathrm{TM}}$ version 20 was used to explore factor properties of the PALS with a random selection of $30 \%$ of the sample. The random selection was conducted in SPSS, and the remaining $70 \%$ of the sample was used for the confirmatory factor analysis. AMOS software was used for conducting confirmatory factor analysis of the factor structure observed in the PAF. Polychoric correlations with the PALS CFA factors and other measures of pet attachment were conducted in order to establish convergent validity. To evaluate the proximal (versus distal) relationships that participants had with their pets, and how the proximal nature of the relationship may relate to attachment to pets, we conducted a MANOVA with linear contrasts for each subscale; the independent variable was recency of having lived with a pet.

\section{Results}

\section{Principle Axis Factoring}

Before conducting analyses, we examined histograms for each of the 39 items on the PALS-39 in order to ensure that none of the 
items had floor or ceiling effects. All items were approximately normally distributed with some items having slight to moderate negative skews. Bartlett's test for sphericity was significant, $\chi^{2}(741)=6002.65, p=.0001$, indicating that there was no violation of the assumption of sphericity. Kaiser-Meyer-Olkin Statistic measuring sampling adequacy was also significant, $\mathrm{KMO}=.95$. These tests indicate that the data set is suitably large and robust for statistically interpretable findings.

The PAF with four factors is presented in Table 1. The promax rotation converged in six iterations. Factor loadings below .30 were suppressed from the output, as is commonly practiced, for clarity. Each factor had eigenvalues $>1.0$, and items loading on each factor had clear themes. Factor $1=$ "Love," eigenvalue $=18.09$, Factor $2=$ "Regulation," eigenvalue $=3.05$, Factor $3=$ "Personal Growth,” eigenvalue $=1.48$, Factor $4=$ "Negative impact," eigenvalue $=1.23$. The total variance explained by these factors was $56.77 \%$.

We examined double factor loadings and weaker factors (low loadings). Four PALS items that had double factor loadings but were small and similar in size (PALS 3, 4, 23, 30), were dropped from the measure because we deemed them to not provide unique information to the substructure of the overall construct of pet attachment or life impact of pets. Three additional items had double loadings (PALS 26, 29,33 ) and the larger of the loadings were used to guide the decision to keep these times with the factor associated with this larger loading. This decision was based on evaluating whether the particular item conceptually fit with that factor of the larger loading and potentially added some unique information.

\section{Confirmatory Factor Analysis.}

In order to confirm the structure of the revised measure, a cross-validation of the EFA solution on the remaining sub-sample was conducted using confirmatory factor analysis (CFA). Four cases had missing data and were removed resulting in a final sample size of 445 . We tested a four-factor model underlying the remaining 35-items (selected as described above). Prior to the CFA, the data were evaluated for multivariate normality using the SPSS macro described in DeCarlo (1997). An omnibus test for multivariate normality based on Small's statistic showed that the distribution of the indicators $\left[\chi^{2}(70)=2107.09, \mathrm{p}<.001\right]$ deviated significantly from a normal distribution. Thus, a robust maximum likelihood estimation method was used for fitting the measurement models. This estimation method analyzes the data using maximum likelihood and robust standard errors. Moreover, the value of $\chi^{2}$ is adjusted by an amount that reflects the magnitude of observed kurtosis, a test known as Satorra-Bentler $\chi^{2}$ (Satorra \& Bentler, 1994).

The sample variance-covariance matrix of the sub-sample was analyzed using EQS 6.1 (Bentler, 2006). Goodness of fit was evaluated using the absolute chi-square test, root mean square error of approximation (RMSEA) and its 90\% confidence interval (90\% CI), and TuckerLewis Index (TLI). Multiple indices were provides because they provide distinct pieces of information about model fit (i.e., statistical fit; absolute fit adjusting for parsimony; incremental fit). Because the chi-square test is highly sensitive to sample size, it is recommended that the decision be based upon additional fit indices (Joreskog \& Sorbom, 1989). Following common guidelines (e.g., Hu \& Bentler, 1999), acceptable model fit was defined by the following criteria: RMSEA $(\leq .08$ adequate fit; $\leq .06,90 \% \mathrm{CI} \leq$ .06 , good fit) and TLI $(\geq .90$ adequate fit; $\geq .95$ good fit). Based upon the EFA pattern matrix, items 2, 36, 8, and 13 were used as marker indicators for Factors 1 - 4, respectively.

Overall fit indices are provided in Table 2. The chi-square test, as expected, was statistically significant $(p<.001)$ suggesting discrepancy 
between model-implied population covariances and the actual observed sample covariance. On the other hand, the model meets suggested TLI (.92) and RMSEA (.05) values for adequate fit. Given the model's reasonable specification (RMSEA) and improvement over the null (TLI), we conclude the four-factor model provides an acceptable approximation of the data. All indicators significantly and reliably loaded on their respective factors (Table 3 provides factor loadings). The inter-correlations of the four factors are as follows: $\phi_{\mathrm{Fac} 1-\mathrm{Fac} 2}=.84$, $\phi_{\text {Fac1-Fac3 }}=.80, \phi_{\text {Fac1-Fac4 }}=.11, \phi_{\text {Fac2-Fac3 }}$ $=.83, \phi_{\mathrm{Fac} 2-\mathrm{Fac} 4}=-.16$, and $\phi_{\mathrm{Fac} 3-\mathrm{Fac} 4}=. .07$. These results indicate the first three factors are somewhat interrelated, with the latent correlation of Factor 1 and Factor 2 being slightly (although not significantly) higher than the remaining factor correlations. The findings also suggest Factor 4 is distinct from the remaining three. These results suggest PALS is best viewed as a multidimensional construct with potential for higher-order factors. In light of the model's adequate fit, future research could refine items and expand content to better represent and model the construct space.

\section{Convergent validity}

\section{PALS with other measures of HAI.}

In order to establish convergent validity with four other measures of HAI, we computed polychoric correlations in R. Table 4 displays these correlations of the PALS subscales and the Anthropomorphism Scale (Albert \& Bulcroft, 1988), the Companion Animal Bonding Scale (CABS; Poresky et al., 1987), the full scale mean and three subscales of the Lexington Attachment to Animals Scale (LAPS; Johnson et al., 1992), and the CENSHARE Pet Attachment Scale (PAS; Holcomb et al., 1985). The first three factors of the PALS had good convergent validity with the other measures of HAI. The fourth factor of the PALS, "Negative Impact," did not correlate consistently with the other measures. This result was expected, given that the factor introduced items unlike those in the positivity-focused items of the other measures. The Negative Impact factor's items were reverse scored, so higher values indicated less negative impact of pet ownership. Overall, the more respondents disagreed with the notion that pets had negatively impacted them, the more they were attached on the LAPS and the more they supported animal rights and welfare (see Table 4 for correlations between measures).

Polychoric correlations between the PALS factors and the Multidimensional Scale of Perceived Social Support (MSPSS; Zimet et al., 1988) are also presented in Table 4. All subscales of the MSPSS were significantly positively correlated with attachment to pets on all measures except the Anthropomorphism Scale. There was no relation between MSPSS subscales and the people-substituting subscale of the LAPS, which indicated that social support from pets may be unrelated to human social support. On the PALS, the MSPSS modestly correlated to finding love in animal relationships and to denying that pets are a burden. Perceived social support from humans did not relate to experiencing pets as regulating one's emotions or as a means of personal growth.

The four factors of the PALS were not all inter-correlated, suggesting some unique aspects to the different factors. Love, Regulation, and Personal Growth all are moderately to highly correlated, but not so highly correlated that they might better be explained as a single factor. Interestingly, Regulation is negatively correlated to Negative Impact whereas Love and Personal Growth are not statistically significantly related to Negative Impact. This finding suggests that the more one uses pets for emotion regulation, the more one endorses Negative Impact (stress) of pet ownership.

A further test of criterion validity was to evaluate whether the PALS was sensitive to 
differences in current relationship benefits or emotional reliance on pets. If the PALS was sensitive to relational attachment, then we expected that current pet owners would report higher scores on the PALS than did former pet owners. The rationale is that attachment relationships provide some emotional regulation benefits, and the PALS is written in the present tense. We tested this hypothesis with a MANOVA using a dichotomous independent variable of current pet owner or former pet owner and the four subscales of the PALS as the dependent variables. The omnibus MANOVA was significant, $F(4,625)=9.30$, Wilks' Lambda $=.94$, partial $\eta^{2}=.056$. Univariate tests were statistically significant for the Love, Regulation, and Personal Growth subscales but not the Negative Impact Scale. These results were: Love, $F(1,628)=32.04, p=.0001$, partial $\eta^{2}=.05$; Regulation, $F(1,628)=10.93, p=$ .001 , partial $\eta^{2}=.017$; Personal Growth $F$ $(1,628)=10.78, p=.001$, partial $\eta^{2}=.017$; Negative Impact $F(1,628)=3.49, p=.062$, partial $\eta^{2}=.005$, where those who were current pet owners in the study had higher scores on Love, Regulation, and Personal Growth subscales of the PALS. Current pet owners also endorsed higher Negative Impact scores than did former pet owners, but this difference was not statistically different.

Individual differences in pet attachment.

A 2 (gender) x 7 (pet type) MANOVA with the four subscales of the PALS as the dependent variables revealed a significant main effect for pet type where dog owners scored the highest and fish owners scored the lowest. The main effect for gender was not significant, however observed power was .33. When two MANOVAs were conducted with gender and pet type as the independent variables, the omnibus MANOVAs were statistically significant for both. Chi-square tests of independence revealed that males and females owned statistically equivalent numbers of dogs, birds, reptiles, fish, rodents, and farm animals (all $p \mathrm{~s}>.05$ ). More females $(n=54)$ than males $(n=28)$ in the sample owned cats, $\chi^{2}=8.24, p<.01$. We then recoded the seven-level pet type variable into a new three-level variable (dog, cat, other), in order to meet equal variances assumption. We conducted a 2 (gender) x 3 (pet type) MANOVA to test for gender $x$ pet type interactions. The omnibus MANOVA was statistically significant for pet type and gender, but there was not a statistically significant interaction $(p=.60)$. For pet type, Wilks' Lambda $=.82, F(8,1188)=$ 14.62, $p=.0001$, partial $\eta^{2}=.09$, and for gender, Wilks' Lambda $=.98, F(4,593)=3.46$, $p=.008$, partial $\eta^{2}=.02$. The results of the univariate tests for differences between pet types were all statistically significant expect for the Negative Impact factor. These were: Love, $F(2$, 596) $=$ 55.27, $p=.0001$, partial $\eta^{2}=.16$; Regulation, $F(2,596)=39.90, p=.0001$, partial $\eta^{2}=.12$; Personal Growth, $F(2,596)=19.70, p$ $=.0001$, partial $\eta^{2}=.06$; Negative impact, $F(2$, 596) $=1.57, p=.209$, partial $\eta^{2}=.01$. Bonferroni pairwise comparisons were statistically significant for Love and Regulation where scores for owners of dogs $>$ cats $>$ other. On the Personal Growth factor, dog owners indicated statistically significant higher values than did owners of cats and other animals; cat and other pet owners did not differ in rating Personal Growth benefits. See Table 5 for means and SDs.

As predicted, there was a significant difference in pet attachment between males and females for all of the factors except negative impact: Love, $F(1,596)=13.77, p=.001$, partial $\eta^{2}=.02$; Regulation, $F(1,596)=7.62, p$ $=.006$, partial $\eta^{2}=.013$; Personal Growth, $F(1$, 596) $=5.90, p=.015$, partial $\eta^{2}=.01$; Negative impact, $F(1,596)=.15, p=.697$, partial $\eta^{2}=$ .0001. Females had higher scores than did males on the Love, Regulation, and Personal Growth factors of the PALS. 


\section{Discussion}

Pet ownership and attachment are ubiquitous in Western culture. A common belief is that pet ownership and interaction with animals is beneficial for humans (Allen, 2003; Herzog, 2011). The current study sought to address measurement limitations related to pet attachment and to elucidate new aspects of pet attachment. Our goal was to refine and validate a qualitatively derived measure of attachment to pets which would measure positive and negative aspects of pet ownership and attachment. We used other published measures of pet attachment to validate the current measure and, based on the literature review, included a measure of human social support.

We also evaluated whether pet attachment was an attributional or relational concept. If it was attributional, (i.e., based on beliefs or cognitions) then attachment was expected to be similar across types of pets and to be stable regardless of whether one currently did or did not have a pet. In contrast, if pet attachment was relational, then we expected to see that current pet owners would have higher scores on the PALS than would former pet owners.

There are numerous measures of pet attachment (see Anderson, 2007) and none has been distinguished as a "gold standard." Studies have had inconsistent results (Herzog, 2011) at least in part due to measurement issues. In the development of the PALS, we used theory, the literature, and importantly, participant feedback to create a comprehensive measure of pet attachment. We collected data at three different universities as we developed items, and in this article we provide a four-factor measure of pet attachment and life impact that accounted for $56.77 \%$ of the variance in the sample. A positive quality of the PALS is that it is broad in scope and introduces new aspects of pet ownership that may shed light on the benefits of HAI.
The correlations between the factors of the PALS support the notion that there are multiple aspects of pet attachment. Love, Regulation, and Personal Growth were all moderately to strongly correlated but these correlations were not high enough to suggest redundancy. The Negative Impact subscale was only negatively related to Regulation, suggesting that individuals who use pets for emotion regulation are also endorsing more stress from ownership. This negative correlation may offer some insight into why individuals who find pet ownership stressful or a financial burden continue to own pets; perhaps cognitive dissonance or a belief that the stress is 'worth it' offsets the costs. This negative correlation is modest, so replication of the findings is important before putting too much weight on this theorizing.

The Love, Regulation, and Personal Growth factors of the PALS moderately to strongly correlated to the Anthropomorphism, LAPS, CABS, and PAS scales. These correlations establish good convergent validity for the PALS. It is notable that the Negative Impact factor of the PALS was related to attachment and animal rights/welfare of the LAPS. The more attached and the more one believed in animal rights/welfare, the more one was willing to spend/sacrifice for a pet. The aspect of Negative Impact and stressors related to pets is important for future research. In the future, we hope to examine whether having pets that require more maintenance or that place greater restrictions on one's life could relate to more stressors, and whether those stressors are offset by more perceived benefits.

There have been inconsistent results in the literature about whether social support is related to various measures of HAI (Herzog, 2011). This inconsistency may be due to the fact that some tests of HAI are insufficiently measuring aspects of relationship per se. Social support may not be related to the overarching construct of HAI but only to relational aspects of pet 
attachment. This notion was supported in the current study. The subscales of the perceived social support scale (MSPSS) were weakly and positively correlated to Love and Negative impact (reverse scored) and were not related to Regulation or Personal Growth. Interestingly, social support was correlated to general attachment and animal rights and welfare of the LAPS but was not significantly correlated to the people substitution scale of the LAPS. Whether this suggests poor construct reliability of that particular subscale of the LAPS is also a question for future research. Nonetheless, social support may be a way of discriminating between distinct constructs of pet attachment and should be pursued in future research.

In order to evaluate whether the PALS was measuring an attitude/belief about pets or whether it was measuring attachment, we queried the proximal nature of relationship with pets. We compared current pet owners to former pet owners on the four subscales of the PALS. Given that there were differences on Love, Regulation, and Personal Growth, our findings suggest that the PALS is sensitive to relational aspects that are fulfilled in pet relationships in the current context. This finding suggests that the PALS may be sensitive to changes in humans' relationships with pets, and this sensitivity will be an important question for future study. This finding adds construct validity to the PALS as a measure of attachment to pets rather than a measure of beliefs about benefits of pets or attitudes towards humananimal relationships in general. If the PALS measured only attitudes towards pets, as other measures do, it would not be sensitive to differences between current pet owners and former owners. Hence, the PALS may be useful for measuring emotional salience and current reliance on pets for emotional needs.

One of the goals of the current study was to delineate individual differences in pet attachment at the levels of pet owner (gender) and type of pet. In examining individual differences within the current sample, we conducted chi-square tests in order to determine whether type of animal that participants were attached to was associated with gender. For cats only, a greater proportion of respondents were female. Nonetheless, there was no gender by pet type interaction for attachment to pets on the PALS. It is important to note, however, that respondents were not asked whether they chose the particular pet to which they were attached and that family pets may be chosen by other household members. Hence, examining differences in gender and type of pet may be more effective at the point of pet adoption.

As expected, there was a main effect for gender, with females having higher scores than males on Love, Regulation, and Personal Growth factors of the PALS. Because of the inconsistent gender differences in the literature, future studies that explore who benefits from interactions with animals should consider splitting files by gender for data analysis. It is notable in this sample that males and females were no different on the Negative Impact factor. While females feel more love from their pets, use the pets more for emotional regulation, and have had more personal or emotional growth from their pets, both males and females equally deny that having a pet is stressful, is a financial hardship, or has negatively impacted them. Thus, from an individual differences perspective, variance appears to be attributed to degrees of benefits of having a pet rather than to degrees of costs of having a pet. Nevertheless, querying negativity may shed light on who benefits from having a pet.

There was also a main effect for type of pet, with dog owners having higher scores on the PALS than cat owners and cat owners having higher scores than owners of other types of pets. This may be because of social support aspects and anthropomorphizing aspects of relationships with dogs over other types of animals 
(Antonacopoulos \& Pychyl, 2010). Reasons for these differences are only speculative at this point.

\section{Conclusions, Limitations, and Future Directions}

Herzog (2011) identified numerous limitations in the important developing research area of HAI. In the present study, we sought to address some of the weaknesses in the literature. The current study participants were motivated to participate for research credit rather than selfselecting to a study that might confirm a belief that pets are beneficial. We also used a large sample that was more heterogeneous with regard to pet ownership than previous studies. We introduce the first qualitatively developed measure using textual analysis of pet attachment that covers a spectrum of aspects of costs and benefits. The measure converged well with other measures of pet attachment. The factors of the PALS appeared to provide some unique measurement aspects that were not represented by other measures of pet attachment. We also replicated other research that found females to be slightly more attached to pets than males and dog owners to be more attached than cat owners. We extend previous research by evidencing that cat owners were more attached than owners of other kinds of pets (e.g., fish, hamsters, or rabbits), and that from a costs perspective, there are not individual differences in terms of who experiences negatives to pet ownership. The latter point is important for considering viability of random assignment to having pets for future intervention work (allergies and other medical reasons not withstanding). We extended past research by evidencing that attachment to pets is a relational concept rather than an attitude or ideology.

The current research was limited in age and educational level of participants. Future research would benefit from stratified samples of participants in order to explore aspects of socio-economic stress that could relate to pet attachment. Also, we may further gain understanding of attachment to pets by examining why individuals who have lived with pets in the past no longer live with pets. The PALS is also limited in that it is a self-report measure. We hope, in future research, to validate the self-report with physiological data (cortisol, heart rate, blood pressure) when in a pet's presence. Our sample was also limited to college students, many of whom live in dormitories or other rental housing that does not allow pets of any sort. In the future, this limitation should be further explored when examining students' transition to college and recency of living with a pet.

We hope that the PALS can be used to inform additional theory about HAI. This study with the PALS suggests that we can use a selfreport measure to capture some aspect of attachment and current relationships with pets. This is important to distinguish emotions and attachment from general beliefs about the values and benefits of pets. The current investigation suggests that the notion of using attachment theory is appropriate for HAI and that we could continue to draw on this theory for considering the nature of human-pet relationships. We hope that the PALS can continue to be developed to incorporate other attachment based concepts for example, keeping an attachment figure in close proximity could be beneficial (Bowlby, 1982). By using this theory, and continuing work in this field, we may be able to observe the possible bidirectional nature of an attachment relationship between humans and their pets. While animals cannot complete measures, we may be able to develop observational paradigms in which we can determine whether pets or humans seek or benefit from proximity, comfort, and love from each other. In this regard, we may even find, as with humans, that there is attachment security and insecurity in human-animal relationships. 
The substantial media attention to the benefits of pets and human-animal interactions has brought a call for more research (Kruger, Symme, \& Serpell, 2004). Two significant gaps in the literature are Who receives health benefits from human animal interaction and who does not? The second major gap is that we do not understand what aspects of HAI produce the observed health benefits. Our hope is that multifactorial pet attachment instrument (PALS) presented in this manuscript, and examination of several individual difference factors (gender, type of pet) will help identify key independent variables to be examined in future research. We also hope that future research would study attachment in human-pet interactions over time. Examining how attached one feels to a pet within the first week of ownership, after a year, and in subsequent years, could potentially inform the attachment specific aspects of understanding the nature of HAI.

\section{References}

Ainsworth, M. D. S. (1991). Attachments and other affectional bonds across the life cycle. In C. M. Parkes, J. StevensonHinde and P. Marris (Eds.), Attachment Across the Lifecycle (pp. 33-51). New York, NY: Routledge.

Albert, A. \& Bulcroft, K. (1988). Pets, families, and the life course. Journal of Marriage \& the Family, 50(2), 543-552.

Allen, K. (2003). Are pets a healthy pleasure? The Influence of pets on blood pressure. Current Directions in Psychological Science, 12(6), 236-239.

Allen, K., Shykoff, B. E., \& Izzo, J. L., Jr. (2001). Pet ownership, but not ACE inhibitor therapy, blunts home blood pressure responses to mental stress. Hypertension, 38(4), 815-820.

American Veterinary Medical Association. (2006). U.S. pet ownership and demographics sourcebook. Retrieved from http://www.avma.org/reference/marketstat s/sourcebook.asp

Anderson, D.C. (2007). Assessing the humananimal bond: A compendium of actual measures. West Lafayette, IN: Purdue University.

Antonacopoulos, N. M. D., \& Pychyl, T. A. (2008). An examination of the relations between social support, anthropomorphism and stress among dog owners. Anthrozoös, 21(2), 139-152.

Antonacopoulos, N. M. D., \& Pychyl, T. A. (2010). An examination of the potential role of pet ownership, human social support and pet attachment in the psychological health of individuals living alone. Anthrozoös, 23(1), 37-54.

Bentler, P. M. (2006). EQS 6 structural equations program manual. Encino, CA: Multivariate Software, Inc.

Bowlby, J. (1982). Attachment (Vol. 2). New York, NY: Basic Books.

Cohen, S. P. (2002). Can pets function as family members? Western Journal of Nursing Research, 24(6), 621-638.

Crawford, E. K., Worsham, N. L., \& Swinehart, E. R. (2006). Benefits derived from companion animals, and the use of the term 'attachment.' Anthrozoös, 19(2), 98112.

Cromer, L. D., \& Freyd, J. J. (2004). Stuffed animals, pets and dissociation revisited. Paper presented at the American Association for the Advancement of Science, Annual Conference: Seattle, WA.

DeCarlo, L. T. (1997). On the meaning and use of kurtosis. Psychological Methods, 2(3), 292-307.

Friedmann, E., Thomas, S. A., Wilson, C. C., \& Turner, D. C. (1995). Pet ownership, social support, and one-year survival after acute myocardial infarction in the Cardiac Arrhythmia Suppression Trial (CAST). In C. Wilson \& D. Turner (Eds.), Companion 
animals in human health (pp. 187-201). Thousand Oaks, CA: Sage Publications.

Headey, B. (1999). Health benefits and health cost savings due to pets: Preliminary estimates from an Australian national survey. Social Indicators Research, 47(2), 233-243.

Herzog, H. (2007). Gender differences in human-animal interactions: A review. Anthrozoös, 20(1), 7-21.

Herzog, H. (2011). Impact of pets on human health and psychological well-being: Fact, fiction, or hypothesis? Current Directions in Psychological Science, 20(4), 236-239.

Holcomb, R., Williams, C. R., \& Richards, S. P. (1985). The elements of attachment: Relationship maintenance and intimacy. Journal of the Delta Society, 2(1), 28 -34.

Hu, L., \& Bentler, P. M. (1999). Cutoff criteria for fit indexes in covariance structure analysis: Conventional criteria versus new alternatives. Structural Equation Modeling: A Multidisciplinary Journal, 6(1), 1-55.

Johnson, T. P., Garrity, T. F., \& Stallones, L. (1992). Psychometric evaluation of the Lexington Attachment to Pets Scale (LAPS). Anthrozoös, 5(3), 160-175.

Joreskog, K. D., \& Sorbom, D. (1989). LISREL VII user's guide. Mooreseville, IN: Scientific Software, Inc.

Kruger, K.A., Symme, W.T., \& Serpell, J.A. (2004). Can animals help humans heal? Animal-assisted interventions in adolescent mental health. [White paper]. Retrieved from

http://research.vet.upenn.edu/Portals/36/m edia/CIAS_AAI_white_paper.pdf

Kurdek, L. A. (2009). Young adults' attachment to pet dogs: Findings from open-ended methods. Anthrozoös, 22(4), 359-369.

Morovati, D. R., Steinberg, A. L., Taylor, L. C., \& Lee, H. B. (2008). Further validation evidence for the Pet Attitude Scale. North
American Journal of Psychology, 10(3), 543-552.

Nagasawa, M., Mogi, K., \& Kikusui, T. (2009). Attachment between humans and dogs. Japanese Psychological Research, 51(3), 209-221.

Nicoll, K., Trifone, C., \& Samuels, W. E. (2008). An in-class, humane education program can improve young students' attitudes toward animals. Society and Animals, 16(1), 45-60.

Poresky, R. H., Hendrix, C., Mosier, J. E., \& Samuelson, M. L. (1987). The Companion Animal Bonding Scale: Internal reliability and construct validity. Psychological Reports, 60(3), 743-746.

Rowan, A. N., \& Beck, A. M. (1994). The health benefits of human-animal interactions. Anthrozoös, 7(2), 85-89.

Satorra, A., \& Bentler, P. M. (1994). Corrections to test statistics and standard errors in covariance structure analysis. In A. von Eye \& C. C. Clogg (Eds.), Latent variable analysis: Applications for developmental research (pp. 399 - 419). Thousand Oaks, CA: Sage.

Sable, P. (1995). Pets, attachment, and wellbeing across the life-cycle. Social Work, 40(3), 334-341.

Siegel, J.M., Angulo, F.J., Detels, R., Wesch, J., \& Mullen, A. (1999). AIDS diagnosis and depression in the multicenter AIDS cohort study: The ameliorating impact of pet ownership. AIDS Care, 11(2), 157-170.

Staats, S., Sears, K., \& Pierfelice, L. (2006). Teachers' pets and why they have them: An Investigation of the human animal bond. Journal of Applied Social Psychology, 36(8), 1881-1891.

Staats, S., Wallace, H, \& Anderson, T. (2008). Reasons for companion animal guardianship (pet ownership) from two populations. Society and Animals, 16(3), 279-291. 
Stammbach, K. B., \& Turner, D. C. (1999). Understanding the human-cat relationship: Human social support or attachment. Anthrozoös, 12(3), 162-168.

Winefield, H. R., Black, A., \& Chur-Hansen, A. (2008). Health effects of ownership of and attachment to companion animals in an older population. International Journal of Behavioral Medicine, 15(4), 303-310.

Zilcha-Mano, S., Mikulincer, M., \& Shaver, P. R. (2011). An attachment perspective on human-pet relationships: Conceptualization and assessment of pet attachment orientations. Journal of Research in Personality, 45(4), 345-357.

Zimet, G.D., Dahlem, N.W., Zimet, S.G., \& Farley, G.K. (1988). The multidimensional scale of perceived social support. Journal of Personality Assessment, 52(1), 30-41. 
Table 1

Principle Axis Factoring, Four-Factor Model Loadings

\begin{tabular}{|c|c|c|c|c|c|}
\hline Item & & F1 & F2 & F3 & F4 \\
\hline 1. & PALS 2 & .72 & & & \\
\hline 2. & PALS 19 & .72 & & & \\
\hline 3. & PALS 32 & .69 & & & \\
\hline 4. & PALS 10 & .69 & & & \\
\hline 5. & PALS 22 & .68 & & & \\
\hline 6. & PALS 27 & .66 & & & \\
\hline 7. & PALS 15 & .66 & & & \\
\hline 8. & PALS 11 & .65 & & & \\
\hline 9. & PALS 17 & .65 & & & \\
\hline 10. & PALS 5 & .64 & & & \\
\hline 11. & PALS 16 & .61 & & & \\
\hline 12. & PALS 12 & .60 & & & \\
\hline 13. & PALS 24 & .58 & & & \\
\hline 14. & PALS 14 & .55 & & & \\
\hline 15. & PALS 29 & .53 & .34 & & \\
\hline 16. & PALS 20 & .47 & & & \\
\hline 17. & PALS 21 & .45 & & & \\
\hline 18. & PALS 4 & .43 & & .32 & \\
\hline 19. & PALS 3 & .39 & & .35 & \\
\hline 20. & PALS 30 & .36 & .35 & & \\
\hline 21. & PALS 36 & & .76 & & \\
\hline 22. & PALS 35 & & .70 & & \\
\hline 23. & PALS 34 & & .62 & & \\
\hline 24. & PALS 37 & & .58 & & \\
\hline 25. & PALS 25 & & .56 & .34 & \\
\hline 26. & PALS 38 & & .49 & & \\
\hline 27. & PALS 26 & .42 & .48 & & \\
\hline 28. & PALS 23 & & .47 & .35 & \\
\hline 29. & PALS 39 & & .47 & & \\
\hline 30. & PALS 28 & & .33 & & \\
\hline 31. & PALS 8 & & & .66 & \\
\hline 32. & PALS 7 & & & .62 & \\
\hline 33. & PALS 9 & & & .47 & \\
\hline 34. & PALS 1 & & & .33 & \\
\hline 35. & PALS 31 & & & .30 & \\
\hline 36. & PALS 13 & & & & .71 \\
\hline 37. & PALS 18 & & & & .70 \\
\hline 38. & PALS 6 & & & & .53 \\
\hline 39. & PALS 33 & & -.31 & & .49 \\
\hline
\end{tabular}

*Note. Loadings indicate strength of relationship with the identified factor. Where there are multiple factor loadings, the factor loading used in the final version is bolded. Items are presented in order of strength of loadings from highest to lowest for each factor. 
Table 2

Goodness-of-Fit Summary Table for the 35-item PALS Scale

\begin{tabular}{lccccc}
\hline Model & $d f$ & Satorra-Bentler $\chi^{2}$ & RMSEA & $\begin{array}{c}\text { RMSEA 90\% } \\
\text { CI }\end{array}$ & TLI \\
\hline Null Model & 595 & $10464.02^{* * *}$ & .19 & $.190-.196$ & - \\
Four-factor & 554 & $1283.15^{* * *}$ & .05 & $.050-.058$ & .92 \\
\hline
\end{tabular}

Note: $\chi^{2}=$ chi-square, $\mathrm{df}=$ degrees of freedom, TLI $=$ Tucker-Lewis index, RMSEA = root mean square error of approximation with $90 \%$ confidence intervals ${ }^{*} p<05 .{ }^{* *} p<.01 .{ }^{* * *} p<.001$. 
Table 3

Standardized Four-Factor Loadings for 35-Item PALS Final Solution

\begin{tabular}{|c|c|c|c|c|c|}
\hline \multicolumn{2}{|c|}{ Item } & \multicolumn{4}{|c|}{ Four-Factor } \\
\hline & & F1 & $\mathrm{F} 2$ & F3 & $\mathrm{F} 4$ \\
\hline 1. & PALS 2 & .71 & & & \\
\hline 2. & PALS 19 & .78 & & & \\
\hline 3. & PALS 32 & .76 & & & \\
\hline 4. & PALS 10 & .81 & & & \\
\hline 5. & PALS 22 & .78 & & & \\
\hline 6. & PALS 27 & .80 & & & \\
\hline 7. & PALS 15 & .85 & & & \\
\hline 8. & PALS 11 & .84 & & & \\
\hline 9. & PALS 17 & .87 & & & \\
\hline 10. & PALS 5 & .74 & & & \\
\hline 11. & PALS 16 & .80 & & & \\
\hline 12. & PALS 12 & .85 & & & \\
\hline 13. & PALS 24 & .82 & & & \\
\hline 14. & PALS 14 & .79 & & & \\
\hline 15. & PALS 29 & .56 & & & \\
\hline 16. & PALS 20 & .38 & & & \\
\hline 17. & PALS 21 & .60 & & & \\
\hline 18. & PALS 36 & & .86 & & \\
\hline 19. & PALS 35 & & .84 & & \\
\hline 20. & PALS 34 & & .80 & & \\
\hline 21. & PALS 37 & & .74 & & \\
\hline 22. & PALS 25 & & .78 & & \\
\hline 23. & PALS 38 & & .77 & & \\
\hline 24. & PALS 26 & & .76 & & \\
\hline 25. & PALS 39 & & .68 & & \\
\hline 26. & PALS 28 & & .48 & & \\
\hline 27. & PALS 8 & & & .88 & \\
\hline 28. & PALS 7 & & & .82 & \\
\hline 29. & PALS 9 & & & .56 & \\
\hline 30. & PALS 1 & & & .65 & \\
\hline 31. & PALS 31 & & & .62 & \\
\hline 32. & PALS 13 & & & & .73 \\
\hline 33. & PALS 18 & & & & .68 \\
\hline 34. & PALS 6 & & & & .47 \\
\hline 35. & PALS 33 & & & & .52 \\
\hline
\end{tabular}

Note. Loadings indicate the strength of relationship for each item with the identified factor. The final PALS is based on the CFA and is presented with loadings ordered highest to lowest for each factor. 
Table 4

Polychoric Correlations of the PALS subscales with other measures of HAI

\begin{tabular}{|c|c|c|c|c|c|c|c|c|c|c|c|c|}
\hline Measure & 1. & 2. & 3. & 4. & 5. & 6. & 7. & 8. & 9. & 10. & 11. & 12. \\
\hline $\begin{array}{l}\text { 1. MSPSS Sig } \\
\text { Other }\end{array}$ & - & & & & & & & & & & & \\
\hline 2. Family & $.69 * *$ & - & & & & & & & & & & \\
\hline 3. Friends & $.71 * *$ & $.70 * *$ & - & & & & & & & & & \\
\hline 4. Anthro mean & .08 & $.15^{* *}$ & .07 & - & & & & & & & & \\
\hline 5. LAPS GA & $.21 * *$ & $.23^{* *}$ & $.17 * *$ & $.64^{* *}$ & - & & & & & & & \\
\hline 6. LAPS PS & -.01 & 0 & -.03 & $.61^{* *}$ & $.73^{* *}$ & - & & & & & & \\
\hline 7. LAPS AR/AW & $.18^{* *}$ & $.19 * *$ & $.14^{* *}$ & $.66^{* *}$ & $.76^{* *}$ & $.63^{* *}$ & - & & & & & \\
\hline 8. CABS mean & $.14^{* *}$ & $.14^{*}$ & $.13^{*}$ & $.55^{* *}$ & $.59 * *$ & $.50^{* *}$ & $.49 * *$ & - & & & & \\
\hline 9. PAS mean & $-.14^{* *}$ & $-.16^{* *}$ & $-.13^{* *}$ & $-.66^{* *}$ & $-.77 * *$ & $-.66^{* *}$ & $-.62 * *$ & $-.72 * *$ & - & & & \\
\hline 10. PALS L & $.16^{* *}$ & $.18^{* *}$ & $.12 *$ & $.71 * *$ & $.80^{* *}$ & $.66^{* *}$ & $.69 *$ & $.60^{* *}$ & $-.79 * *$ & - & & \\
\hline 11. PALS R & .05 & .08 & .02 & $.64^{* *}$ & $.71^{* *}$ & $.70^{* *}$ & $.59 * *$ & $.54^{* *}$ & $-.71^{* *}$ & $.81^{* *}$ & - & \\
\hline 12. PALS PG & .09 & .10 & .07 & $.60 * *$ & $.68^{* *}$ & $.60^{* *}$ & $.58 * *$ & $.51^{* *}$ & $-.65 * *$ & $.75^{* *}$ & $.77^{* *}$ & - \\
\hline 13. PALS NI & $.20 * *$ & $.21 * *$ & $.20 * *$ & .03 & $11^{* *}$ & -.11 & $.13^{* *}$ & .05 & $-.08 *$ & .08 & $-15^{* *}$ & -.08 \\
\hline
\end{tabular}

Note: ${ }^{*} \mathrm{p}<.05,{ }^{* *} \mathrm{p}<.01,+\mathrm{p}<.001$ 
Table 5

Pet Attachment and Life Impact Scale (PALS)

\begin{tabular}{ccccc} 
& \multicolumn{4}{c}{ M (SD) } \\
\hline Type of Pet & Love & Regulation & Personal Growth & Negative Impact \\
\hline $\begin{array}{c}\text { Dog } \\
(n=160)\end{array}$ & $3.66(.85)$ & $3.02(.89)$ & $3.00(.92)$ & $4.28(.61)$ \\
$\quad \begin{array}{l}\text { Cat } \\
(n=81)\end{array}$ & $3.31(.94)$ & $2.56(.94)$ & $2.65(.94)$ & $4.41(.50)$ \\
$\begin{array}{c}\text { Other } \\
(n=62)\end{array}$ & $2.51(.97)$ & $2.06(.99)$ & $2.32(.94)$ & $4.30(.69)$ \\
$\begin{array}{c}\text { Total } \\
(n=603)\end{array}$ & $3.49(.94)$ & $2.86(.96)$ & $2.88(.95)$ & $4.30(.61)$ \\
\hline
\end{tabular}




\section{Appendix}

Pet Attachment and Life-Impact Questionnaire (PAL)

This questionnaire is for anyone who has lived with a pet. If you have EVER lived with a pet (whether or not you owned it) please indicate how strongly each statement reflects how your pet has impacted your life. If you have lived with more than one pet please respond with your favorite or most important pet in mind. If you choose a past pet, please respond as if the pet currently lives with you.

What kind of animal was your most special/important/favorite pet?

Responses for each question are:

$\begin{array}{ccccc}\text { Not at all } & \text { Somewhat } & \text { Moderately } & \text { Quite a bit } & \text { Very much } \\ 1 & 2 & 3 & 4 & 5\end{array}$

1. Having a pet has helped my health.

2. My pet is part of my family.

3. My pet is more loyal than most people.

4. My pet has the same privileges a family member.

5. A pet completes the family.

6. Having a pet is stressful.

7. I am more affectionate because of my pet.

8. I have learned compassion from my pet.

9. Having a pet has helped me to understand loss and letting go.

10. My pet gives me unconditional love.

11. My pet gives me something to love.

12. My pet gives me something that I can form a close emotional bond with.

13. Having a pet has negatively impacted me emotionally.

14. My pet is my companion.

15. My pet and I have a special relationship.

16. My pet is loyal.

17. My pet provides comfort for me.

18. I am worse off because I have a pet.

19. I like to cuddle with my pet.

20. I like my pet mostly because it is cute.

21. It's worth giving up other things in life in order to have a pet.

22. Pets take a lot of time but it is worth it.

23. My pet teaches me to be more loving.

24. My pet is my friend.

25. My pet teaches me to trust.

26. My pet calms me down.

27. My pet cheers me up.

28. I take my pet with me to visit people.

29. I keep a picture of my pet with me.

30. I am affected by the way others react to my pet. 
31. My pet teaches me responsibility.

32. My pet is fun and entertaining.

33. My pet is a financial hardship.

34. My pet allows me to feel needed.

35. My pet is someone to lean on and be with me when no one else is there for me.

36. My pet provides stability for me.

37. My pet understands me like no one else has.

38. Talking to my pet makes me feel better.

39. My pet offers protection/safety. 